\title{
Introduction to Knowing What We Know: Theory, Meta-analysis and Review Minitrack
}

\author{
Dirk Hovorka \\ University of Sydney \\ dirk.hovorka@sydney.edu.au
}

\author{
Kai Larsen \\ University of Colorado \\ Kai.Larsen@colorado.edu
}

This minitrack is interested in papers that pursue approaches, methods and conceptual papers which will advance the IS field ability to "know what it knows". Our ability to understand, integrate and synthesize the exponentially growing body of scientific literature in the social sciences is hampered by both structural and social problems. These include a lack of standardization for concepts and constructs across the fields and a lack of infrastructure and powerful search and integration tools which would enable researchers to perform meta-theorization. The aim of this minitrack is to engage the disciplinary infrastructures other fields have successfully progressed (e.g. metaBUS, the Human Behavior Project, Medline and the Biological Science Database) that will enable IS to better know what we know.

Research over the last decades has emphasized theory development in IS and other social and behavioral science disciplines. The resulting proliferation of theories and constructs has numerous redundancies, which can be revealed through review, meta-theorization/meta-analysis and interrogation of the theory discourse. Theory ontologies would benefit the disciplines by identifying what we can now research given what we already know. Theory synthesis or integration will inform social and behavioral sciences research with a better understanding of fundamental theories, help organize our theories to be accessible to practice, and increase our understanding of the philosophical commitments represented in their contextualization and use.

The scope of the papers for the minitrack is quite broad, including for example, the development of theory ontologies, approaches to theory integration, and meta-analytic/review approaches to building cumulative theory. We also welcome approaches to the local contextualization of theory where insight is gained into valuable distinctions. Topics of interest in this minitrack include:

- Approaches to theory meta-analysis, integration or aggregation of social and behavioral science theories;

- The theoretical ties between different disciplines (e.g. healthcare and IS, and sustainability science and IS, energy informatics), or parallel the trends in theorizing the same phenomenon;

- Research on ontologies, taxonomies, frameworks, and categorizations of constructs and variables used in system science theories;

- Conceptual papers on an Social Science Infrastructure that would support social sciences;

- Techniques for the extraction of constructs and relationships from published papers;

- The use of natural language processing, data mining, and predictive analytics to better understand and interrogate theories;

- Discussion of the roles of theories used to explain, approaches used to predict (e.g. neural nets and big data), and of theories of understanding; and

- Exploration of the dependencies of constructs and variables; Exploration of the boundaries of theory "domains." 